\title{
USO DO RESÍDUO DE GRANITO ORIUNDO DA SERRARIA E POLIMENTO COMO CORRETIVO E FERTILIZANTE DE SOLOS AGRÍCOLAS
}

\author{
Alexandre Sylvio Vieira Da Costa ${ }^{1}$; Adolf H. Horn²; \\ Guilherme Kangussú Donagemma ${ }^{1}$; Marcelo Barreto Da Silva ${ }^{1}$
}

RESUMO

\begin{abstract}
O crescimento acelerado da população mundial nas últimas décadas promoveu um aumento significativo no processo de industrialização, tendo como conseqüência o surgimento de problemas ambientais. $\mathrm{O}$ aumento populacional mundial requer uma maior produção de alimentos e bens de consumo, além da maior exploração dos recursos naturais. Um dos maiores problemas ambientais da atualidade encontra-se nas atividades industriais através principalmente da produção de resíduos. Este trabalho teve como objetivo avaliar as potencialidades físico-químicas para uso agrícola dos resíduos de serraria e polimento de granito. Foram coletados resíduos de quatro empresas do Espírito Santo. Os materiais foram analisados no laboratório de solos da Univale em Governador Valadares. Foram avaliados a distribuição granulométrica dos materiais, o estado de suspensão, $\mathrm{pH}$, teores de ferro, teores de metais pesados (chumbo e cádmio) e nutrientes (nitrogênio, fósforo, cálcio, magnésio e zinco. Verificou-se que a dinâmica dos resíduos do processamento das rochas ornamentais está intimamente associada a sua granulometria reduzida e elevada superfície de partícula. $\mathrm{O}$ alto nível de ferro pode se tornar um fator limitante ao seu uso no solo sendo necessária a extração deste elemento. A reduzida taxa de suspensão coloidal indica um material de pouca mobilidade no solo sendo necessária a adequação de seu manejo. Os resíduos se mostraram diversificados em relação à composição mineralógica, mas com potencial para serem utilizados na agricultura.
\end{abstract}

Palavras-chave: aterros industriais, agricultura, reaproveitamento, rochas ornamentais

\begin{abstract}
The rapid growth of world population in recent decades caused a significant increase in the industrialization process, resulting in an emergence of environmental problems. The increasing world population requires more food production and consumer, in addition to greater exploitation of natural resources. One of the biggest environmental problems of today is mainly on industrial activities through the production of waste. This study aimed to evaluate the potential physical and chemical waste to agricultural use from sawmills and polishing granite. Waste were collected from four companies of the Espírito Santo, Brazil. The materials were analyzed in soil laboratory of Univale in Governador Valadares. Was evaluated the size distribution of materials, the suspension state, $\mathrm{pH}$, iron content of heavy metals (lead and cadmium) and nutrients (nitrogen, phosphorus, calcium, magnesium and zinc. Was found that the dynamics of the waste processing the natural stone is closely associated with its reduced size and high particle surface. The high level of iron can become a limiting factor to its use in soil being necessary to extract this element. The low rate of colloidal suspension indicates a material low mobility in soil is necessary the adequacy of its management. The waste proved to be diverse in relation to mineralogical composition, but with potential for use in agriculture.
\end{abstract}

Keywords: landfills, agriculture, reuse, ornamental rocks

\section{INTRODUÇÃO}

A qualidade de vida do homem está intimamente relacionada ao meio ambiente e como fator evidente, é amplamente debatido nas diversas áreas da ciência. Botelho (1987) cita que o processo de poluição ambiental observado no Brasil, seja da água, do solo ou da atmosfera, é um aspecto de grande importância, mas relacionado a um problema mais abrangente pois a poluição e a degradação do ambiente, conseqüência das graves condições sociais e econômicas. Estão geralmente associados a outros fatores, decorrentes da falta de planejamento das atividades urbanas e rurais, do desemprego e do empobrecimento da população. Estes fatores interagem reduzindo a qualidade de vida, ocorrendo paralelamente à deterioração dos recursos naturais e dos mecanismos que permitem o homem viver e trabalhar em determinado ambiente. Este conjunto de recursos e mecanismos é conhecido como "sistema vital".

O crescimento acelerado da população mundial nas últimas décadas promoveu um aumento significativo no processo de industrialização, tendo como conseqüência o surgimento de problemas ambientais. $\mathrm{O}$ aumento populacional mundial requer uma maior produção de alimentos e bens de consumo, além da maior exploração dos recursos naturais. Um dos maiores problemas ambientais da atualidade encontrase nas atividades industriais através principalmente da produção de resíduos lançados diretamente em sistemas naturais sem a observação de critérios definidos, com grande potencial poluidor do solo, águas subterrâneas e superficiais e conseqüentemente queda na qualidade 
de vida da população. Mesmo com as novas tecnologias desenvolvidas, em muitos casos torna-se praticamente impossível a eliminação da capacidade poluidora de alguns resíduos visando o seu manejo, a reciclagem e a reutilização de forma harmoniosa com o meio ambiente.

Com este objetivo, as atividades agropecuárias se adequam ao processo com participação importante na reciclagem e integração dos materiais nos sistemas biogeoquímicos, principalmente dos resíduos que possuem nutrientes minerais que podem ser utilizados pelas plantas cultivadas ( King, 1988).

Segundo Souza et al (2002) a maioria dos resíduos urbanos, industriais ou agroindustriais pode apresentar, além dos elementos minerais, outros compostos que podem acarretar sérios riscos à qualidade do meio ambiente (Souza et al., 2002).

A composição química dos resíduos industriais utilizados como matéria prima para insumos agrícolas deve ser freqüentemente monitorada. Este processo é imprescindível para utilização racional e segura destes materiais na agricultura (Fontes, 2001).

A atual tendência é tornar-se cada vez mais dispendiosa para o produtor rural a aquisição de calcários e fertilizantes. Deste modo, o uso de fontes alternativas de resíduos industriais que apresentam qualidade de corretivo do solo e de fertilizantes e que são depositados em pátios, aterros e até mesmo a céu aberto pode ser uma solução, não apenas visando a eliminação do passivo ambiental da indústria, mas também a redução dos custos de produção da agropecuária que beneficiaria toda a cadeia, do produtor ao consumidor.

O estudo apurado dos parâmetros físicos e químicos dos resíduos das industrias de pedras ornamentais e do solo é fundamental para nortear um manejo adequado da aplicação deste resíduo no solo objetivando o uso sustentável da área, reduzindo os riscos de contaminação do meio ambiente e dos recursos hídricos. A caracterização do material nos permite conhecer o seu potencial agrícola, prevendo seus efeitos nas características físicas, químicas e biológicas do solo assim como na nutrição das plantas. Neste estudo, a mineralogia da fração argila e a textura do solo são fundamentais na avaliação da dinâmica dos nutrientes e dos contaminantes do solo (Pinheiro, 2002; Oliveira et al., 2004; Silva, 2004).

A extração de rochas ornamentais vem aumentando significativamente ao longo dos últimos anos visando atender a demanda da construção civil. O Brasil detém grandes reservas de pedras ornamentais de revestimento (granito e mármore) com os mais variados aspectos estéticos. As pedras naturais ornamentais são produtos que competem com os pisos e revestimentos cerâmicos (Moreira et al., 2003). Dentre os estados produtores, o Espírito Santo é responsável por cerca de $80 \%$ da produção nacional de pedras ornamentais. O município de Cachoeiro do Itapemirim responde por $90 \%$ da produção capixaba, sendo este setor mineral a principal atividade econômica da região, suprindo não apenas o mercado nacional, mas também o internacional, como Estados Unidos e países da Europa.

Atrelado ao processo de produção, a geração de resíduos provenientes do corte dos blocos torna-se inevitável gerando entre 20 e $25 \%$ de pó de pedra e outros acessórios como granalha metálica e cal (Moreira et al., 2003), podendo atingir em certas situações, 50\% de todo o material. A presença destes rejeitos, na forma de lama, no ambiente gera um enorme impacto ambiental podendo causar assoreamento de rios, afetando a fauna e a flora da região, causar problemas respiratórios nos seres vivos bem como uma grande poluição visual. A lama quando seca torna-se um resíduo sólido não degradável classificado como resíduo classe III, inerte. No município de Cachoeiro do Itapemirim, ES, estimase que as indústrias produzam cerca de 400 ton/mês de resíduos na forma de lama (Souto et al., 2001). O uso de tecnologia como o uso de fio diamantado no corte das pedras tem minimizado a geração destes resíduos devido a sua maior precisão (Filgueira, 2001) mas, o aumento da capacidade produtiva e de exportação faz com que a quantidade de resíduos produzidos continue elevada. Outro ponto do processo industrial que deve ser levado em consideração é o polimento das pedras. O processo é realizado por polidoras que possuem metais em sua composição e que desgastam durante o polimento. Este resíduo é condicionado juntamente com os resíduos minerais dos cortes das pedras.

O presente trabalho teve por objetivo identificar as características químicas, físicas e mineralógicas dos resíduos produzidos pelas serrarias de pedras ornamentais do Espírito Santo.

\section{MATERIAIS E MÉTODOS}

Este estudo foi desenvolvido no Laboratório de Química e Fertilidade do Solo da Universidade Vale do Rio Doce, Faculdade de Ciências Agrárias em Governador Valadares-MG. Os resíduos das rochas ornamentais de cada uma das empresas são provenientes do corte dos blocos de granito e outros acessórios como granalha metálica e cal, que se encontram na forma de lama. Os resíduos foram coletados nos tanques de depósitos das empresas Marbrasa, Katugram e Limagram. Na Marbrasa foram coletados resíduos de serraria e polimento (separados), na Katugram apenas polimento e na Limagram, polimento e serraria, juntos pois são armazenados no mesmo tanque.

Para o teste de suspensão os materiais foram triturados manualmente até atingir a granulometria de pó. Foram pesados 20,00 gramas de cada material em balança de precisão. Os materiais foram colocados separadamente em provetas com capacidade de $1000 \mathrm{ml}$ e preenchido com este mesmo volume de água. Após a adição da água, as soluções foram homogeneizadas durante 15 minutos utilizando o stirrer e mantidas em repouso. Após os primeiros 10 minutos de repouso foi retirada uma amostra de $10 \mathrm{ml}$ de cada material na parte intermediária da proveta (centro da proveta) 
utilizando uma pipeta com capacidade para $10 \mathrm{ml}$. A solução recolhida na pipeta foi colocada em cápsula de porcelana e conduzida para estufa de secagem onde permaneceu por 72 horas a temperatura constante de $105^{\circ} \mathrm{C}$ para que houvesse a completa eliminação da água, mantendo apenas o resíduo que se encontrava em suspensão. Este procedimento foi repetido durante uma hora com coletas a cada 10 minutos perfazendo um total de 6 amostras retiradas de cada material. Foram agrupadas as coletas realizadas nos primeiros trinta minutos e nos trinta minutos seguintes. O delineamento experimental empregado foi o inteiramente casualizado com três repetições. A quantidade do material residual em suspensão foi determinada em gramas/Litro.

Para a análise de granulometria foram utilizadas peneiras de malha $1,00 \mathrm{~mm} ; 0,250 \mathrm{~mm} ; 0,125 \mathrm{~mm}$; $0,105 \mathrm{~mm} ; 0,088 \mathrm{~mm}$ e $0,044 \mathrm{~mm}$ que ficam sobrepostas da maior para a menor malha. Foram pesados 30 gramas de cada resíduo seco e peneirado e adicionado água $(300 \mathrm{ml})$ seguido de dispersão no STIRRER durante 5 minutos para desagregação das partículas. Após este procedimento a solução dispersa foi transferida para as peneiras seguida de lavagem intensa do recipiente para que todo o material fosse transferido para as peneiras. Após este procedimento foi realizada a lavagem contínua das peneiras utilizando um pissete, visando a passagem das partículas dos materiais pelas peneiras. Após este procedimento as peneiras foram separadas, e o resíduo retido em cada uma delas foi condicionado em cápsulas de porcelana e levada para estufa de secagem a $105^{\circ} \mathrm{C}$ permanecendo ali durante 72 horas visando à retirada completa da água.

O ferro presente no resíduo foi separado por imantação. Foram pesados 20,00 gramas de resíduo seco de cada empresa. O material foi diluído em 300 $\mathrm{mL}$ de água destilada seguido da passagem de um imã. Foram realizadas diversas passagens na solução até a retirada completa do metal. O ferro extraído foi lavado e depositado em cadinhos de porcelada e colocados na estufa a $105^{\circ}$ c para retirada da água. Após 72 horas o material foi pesado e calculado o teor de ferro nos resíduos.

Estes mesmos materiais residuais forma enviados para o laboratório de análises químicas de solos da Universidade Federal de Viçosa para determinação dos teores dos seguintes elementos: Nitrogênio, Fósforo, Cálcio, Magnésio, Zinco, Chumbo e Cádmio.

\section{RESULTADOS E DISCUSSÃO}

Os resíduos das empresas foram selecionados em função do tipo de atividade e processamento das rochas (serraria e polimento) e a forma de depósito nos aterros industriais (depositados separadamente ou unificados). Na Tabela 1 encontram-se os resultados da separação das partículas dos resíduos por diâmetro. Os

Tabela 1.Granulometria dos resíduos de rochas ornamentais

\begin{tabular}{lrrrr} 
& & Polimento & \multicolumn{1}{l}{ Serraria } \\
\hline 1,000 & $0,00 \%$ & $0,00 \%$ & $0,00 \%$ & $0,00 \%$ \\
0,250 & $20,12 \%$ & $6,96 \%$ & $5,21 \%$ & $4,51 \%$ \\
0,125 & $6,48 \%$ & $2,66 \%$ & $8,46 \%$ & $1,95 \%$ \\
0,105 & $2,65 \%$ & $1,50 \%$ & $2,93 \%$ & $0,50 \%$ \\
0,088 & $2,06 \%$ & $1,28 \%$ & $2,11 \%$ & $0,31 \%$ \\
0,044 & $28,92 \%$ & $18,69 \%$ & $33,89 \%$ & $12,83 \%$ \\
Fundo & $39,77 \%$ & $68,91 \%$ & $47,40 \%$ & $79,90 \%$ \\
\hline
\end{tabular}

Obs: média de três repetições.

valores mostraram-se distintos devido principalmente à diversidade da atividade (polimento e serraria) e a forma de deposição dos resíduos. Como era esperado, os resíduos de polimento do granito das empresas Marbrasa e Katugran apresentaram uma maior proporção de partículas com diâmetro inferior a 0,044 mm, com valores de $68,91 \%$ e $79,90 \%$, respectivamente. O resíduo de serraria da empresa Marbrasa e a mistura de resíduo de polimento e serraria da empresa Limagran apresentaram uma menor proporção de partículas mais finas, variando de $39,77 \%$ a $47,40 \%$, respectivamente.
Estes resultados eram esperados porque o processo de polimento é uma etapa de acabamento onde a placa de rocha é tratada de forma mais cuidadosa com a passagem continuada de polidores para uniformização e brilho de sua superfície. A serraria é um processo inicial de corte de blocos e é realizada por serras de ferro com a utilização de abrasivos para facilitação dos cortes. É um processo mais grosseiro com produção reduzida de partículas de rocha mais finas. Apesar desta diferenciação de processos, não foram encontradas partículas minerais de diâmetro superior a $1,00 \mathrm{~mm}$, 
mesmo no resíduo de serraria, indicando que o material pode apresentar uma grande capacidade de reatividade no solo e rápida mineralização.

$\mathrm{Na}$ Tabela 2, verifica-se que os resíduos apresentam pH em água superior a 7,0 com os resíduos da empresa Marbrasa próximo a 9,0. Estas características químicas dos materiais são importantes no metabolismo químico do solo, pois com o aumento do $\mathrm{pH}$ do solo, a acidez potencial é reduzida, o alumínio tóxico passa para forma insolúvel e não absorvido pelas plantas, a maioria das cargas elétricas do solo (CTC) ficam disponíveis para a adsorção dos nutrientes minerais, reduzindo as perdas por lixiviação e aumentando a eficiência da absorção pelas plantas que apresentarão maior desenvolvimento do seu sistema radicular. A maioria dos macronutrientes ficará na forma disponível na solução do solo, na forma iônica, prontamente disponível para absorção pelas raízes.

Tabela 2. pH dos resíduos de rochas ornamentais (diluição 1:2,5 em água)

\begin{tabular}{ccccc}
\hline & \multirow{2}{*}{$\begin{array}{c}\text { Limagran } \\
\text { (Polimento + serraria) }\end{array}$} & \multicolumn{2}{c}{ Marbrasa } & \multirow{2}{*}{$\begin{array}{c}\text { Katugran } \\
\text { (Polimento) }\end{array}$} \\
\cline { 3 - 4 } & 7,61 & Polimento & Serraria & \\
\hline $\mathrm{pH}$ & & 8,80 & 9,01 & 8,56 \\
\hline
\end{tabular}

O teor de ferro presente nos resíduos (Tabela 3) mostra-se elevado, atingindo valores acima de $14 \%$ nos resíduos de serraria devido à presença de resíduo de granalha. Este metal também foi detectado nos resíduos de polimento que não utiliza granalha, apesar dos valores serem inferiores aos encontrados nos resíduos de serraria, podendo ser proveniente dos polidores que são de origem mineral. $\mathrm{O}$ ferro é considerado um micronutriente essencial, mas em quantidades elevadas pode causar a toxidez das plantas e comprometendo a absorção de outros nutrientes.

$\mathrm{Na}$ Tabela 4, observa-se a quantidade do resíduo de granito que permaneceram em suspensão no período de uma hora. Este ensaio é importante pois tem como objetivo verificar a capacidade de translocação dos resíduos minerais juntamente com a solução do solo pelos macroporos em função de sua característica de suspensão. Os resíduos oriundos do polimento, apesar de apresentarem partículas de menor diâmetro, permaneceram em suspensão em menor proporção quando comparado aos resíduos oriundos da serraria e serraria+polimento das empresas Marbrasa e Limagran, respectivamente. Este fato ocorreu provavelmente pela aglutinação (floculação) das partículas menores que estavam em suspensão devido à presença de cargas elétricas superficiais presentes em quantidades superiores nas partículas coloidais. Esta ligação formou uma estrutura granular maior e mais pesada que promovendo a sua precipitação na solução. No resíduo de polimento da empresa Marbrasa a precipitação das partículas nos primeiros 30 minutos avaliados, comparado aos 30 minutos seguintes foi de aproximadamente $90 \%$, reduzindo de $0,517 \mathrm{~g} / \mathrm{L}$ para $0,050 \mathrm{~g} / \mathrm{L}$. No resíduo de serraria desta mesma empresa, a precipitação foi menor com valores em torno de $60 \%$.

$\mathrm{Na}$ Tabela 5, encontram-se os teores dos nutrientes e dos metais pesados presentes nos resíduos. Em termos de contaminação por chumbo, este metal encontra-se em concentrações muito reduzidas com riscos reduzidos de contaminação do solo e da planta com o manejo adequado do resíduo. Em relação ao cádmio, não foram detectados concentrações acima de 1,0 ppm. Dentre os nutrientes, o nitrogênio praticamente não apareceu em nenhuma amostra, com exceção do resíduo de serraria

Tabela 3. Teor de Ferro dos resíduos de rochas ornamentais (extraídos por imantação em suspensão aquosa)

\begin{tabular}{lcccc}
\hline & \multirow{2}{*}{$\begin{array}{c}\text { Limagran } \\
\text { (Polimento + serraria) }\end{array}$} & \multicolumn{2}{c}{ Marbrasa } & \multirow{2}{*}{$\begin{array}{l}\text { Katugran } \\
\text { (Polimento) }\end{array}$} \\
\cline { 3 - 4 } & & Polimento & Serraria & \\
\hline Ferro (\%) & $9,840 \%$ & $4,647 \%$ & $14,534 \%$ & $3,930 \%$ \\
\hline
\end{tabular}

Tabela 4. Resíduos de rochas ornamentais em suspensão em água nos 30 minutos iniciais e 30 minutos seguintes (coletas realizadas a cada 10 minutos) em gramas por litro $(\mathrm{g} / \mathrm{L})$.

\begin{tabular}{|c|c|c|c|c|}
\hline \multirow{2}{*}{ Coletas } & \multirow{2}{*}{$\begin{array}{c}\text { Limagran } \\
\text { (Polimento + serraria) }\end{array}$} & \multicolumn{2}{|c|}{ Marbrasa } & \multirow{2}{*}{$\begin{array}{l}\text { Katugran } \\
\text { (Polimento) }\end{array}$} \\
\hline & & Polimento & Serraria & \\
\hline 30 minutos iniciais & 0,994 & 0,517 & 4,267 & 0,270 \\
\hline 30 minutos seguintes & 0,630 & 0,050 & 1,727 & 0,150 \\
\hline
\end{tabular}


Tabela 5. Teor total de nutrientes minerais e metais nos resíduos de rochas ornamentais

\begin{tabular}{|c|c|c|c|c|}
\hline & \multirow{2}{*}{$\begin{array}{c}\text { Limagran } \\
\text { (Polimento + serraria) }\end{array}$} & \multicolumn{2}{|c|}{ Marbrasa } & \multirow{2}{*}{$\begin{array}{l}\text { Katugran } \\
\text { (Polimento) }\end{array}$} \\
\hline & & Polimento & Serraria & \\
\hline $\mathrm{N}(\%)$ & - & - & 0,02 & - \\
\hline P (\%) & 0,87 & 0,38 & 0,25 & 0,30 \\
\hline $\mathrm{Ca}(\%)$ & 9,20 & 1,52 & 1,27 & 1,50 \\
\hline $\operatorname{Mg}(\%)$ & 1,30 & 0,68 & 3,92 & 4,56 \\
\hline $\mathrm{Zn}(\mathrm{mg} / \mathrm{kg})$ & 12,70 & 33,80 & 6,20 & 77,80 \\
\hline $\mathrm{Pb}(\mathrm{mg} / \mathrm{kg})$ & 5,50 & 4,00 & 2,40 & 3,90 \\
\hline $\mathrm{Cd}(\mathrm{mg} / \mathrm{kg})$ & - & - & - & - \\
\hline
\end{tabular}

da empresa Marbrasa onde pode ter ocorrido algum tipo de contaminação com matéria orgânica, já que o aterro industrial está localizado a céu aberto. A quase totalidade das rochas não apresenta nitrogênio em sua composição, sendo o seu principal reservatório o ar atmosférico. Em relação ao fósforo, destaque para o resíduo da empresa Limagran (polimento+serraria) com quase $1 \%$ e os resíduos de polimento com valores em torno de $0,35 \%$. Nos teores de magnésio, destaque para os resíduos das empresas Katugran (polimento) e Marbrasa (serraria) com valores em torno de 4\%. A empresa Limagran (polimento+serraria) destacou-se em relação à concentração de cálcio com $9,2 \%$ enquanto os demais resíduos apresentaram concentrações de cálcio em torno de 1,5\%. Estas diferenças provem do tipo de rocha que é serrada e polida e de sua composição mineralógica, além das quantidades de produtos químicos utilizados o processo de produção das placas de rochas ornamentais, principalmente a cal, rica em cálcio. Outro aspecto importante é a solubilidade dos nutrientes. Apesar da presença elevada de alguns nutrientes nos resíduos, se os mesmos não estiverem disponíveis, não poderão ser aproveitados de imediato pelas plantas.

\section{CONCLUSÕES}

De acordo com os resultados obtidos podemos concluir que a dinâmica dos resíduos do processamento de granito está intimamente associada a sua granulometria reduzida e elevada superfície de partícula. $\mathrm{O}$ alto nível de ferro pode se tornar um fator limitante ao seu uso no solo sendo necessária a extração deste elemento. A reduzida taxa de suspensão coloidal indica um material de pouca mobilidade no solo sendo necessária a adequação de seu manejo. Os resíduos se mostraram diversificados em relação à composição mineralógica, mas com potencial para serem utilizados como fertilizantes.

\section{REFERÊNCIAS}

BOTELHO, C. G. 1987. Controle de poluição de áreas rurais. Lavras, $1^{\mathrm{a}}$ ed., Departamento de Engenharia, UFLA. 319 p.

FILGUEIRA, M. 2001. Produção de fios diamantados. Tese de Doutorado, Universidade Estadual do Norte Fluminense, PPGECM-CCT, Tese de Doutorado, 157p.

FONTES, R. L. F. 2001. Avaliação da eficiência de material calcário de origem industrial (Usiminas) como corretivo da acidez do solo para agricultura. Relatório Técnico. UFV, Relatório Técnico, $40 \mathrm{p}$.

KING, L. D. 1988. Retention of metals by several soils of the Southeastern United States. J. Environ. Qual., 17(2):239-46.

MOREIRA, J. M. S.; Freire, M. N.; Holanda, J. N. F. 2003. Utilização de resíduo de serragem de granito proveniente do estado do Espírito Santo em cerâmica vermelha. Cerâmica, 49:262-267.

OLIVEIRA, E. M. M.; Ruiz, H. A.; Ferreira, P. A.; Alvarez, V. V. H.; Borges Junior, J. C. F. 2004. Fatores de retardamento e coeficientes de dispersão difusão de fosfato, potássio e amônio em solos de Minas Gerais. Eng. Agric. Amb., 8:196-203.

PINHEIRO, R. B. 2002. Mobilidade de nitrato em resposta a propriedades eletroquímicas de solos com carga variável. Universidade Federal de Viçosa, Tese de Mestrado, 76 p.

SILVA, A. T. 2004. Movimentação de amônio, nitrato, potássio e fósforo aplicados por fertirrigação em Latossolos.. UFV, Tese de mestrado, $58 \mathrm{p}$.

SOUTO, K. M.; Neves, G. A.; Ferreira, H. C.; Silva, M. C. 2001. Aproveitamento de resíduo de serragem de granitos para confecção de tijolos e telhas cerâmicas. Anais do $45^{\circ}$ Congresso Brasileiro de Cerâmica, Florianópolis, SC, p.701-12. (CDROOM).

SOUZA, C. M. DE; SANTOS, J. R. DOS; SANTOS, J. R. 2002. Caracterização da escória da Bermix do Brasil para uso agrícola. Universidade Federal de Viçosa, Relatório Técnico, 17 p. 